\title{
Thermal Design of Large-Power Focal Plane Components for a Microsatellite Based on Pyrolytic Graphite Sheet
}

\author{
Xiaoguang Xie $\mathbb{D}^{1},{ }^{1,2}$ Guang Jin, ${ }^{1}$ and Minglin $X u^{1}$ \\ ${ }^{1}$ Changchun Institute of Optics, Fine Mechanics and Physics, Chinese Academy of Sciences, Changchun 130033, China \\ ${ }^{2}$ University of Chinese Academy of Sciences, Beijing 100059, China \\ Correspondence should be addressed to Xiaoguang Xie; 7801046ab@163.com
}

Received 20 May 2018; Revised 26 October 2018; Accepted 29 November 2018; Published 25 February 2019

Academic Editor: Jeremy Straub

Copyright (C) 2019 Xiaoguang Xie et al. This is an open access article distributed under the Creative Commons Attribution License, which permits unrestricted use, distribution, and reproduction in any medium, provided the original work is properly cited.

\begin{abstract}
A high-resolution microsatellite's focal plane components have high heat flux and a long working time. In order to solve the heat-dissipation problem of a microsatellite's focal plane components under the condition of narrow space, this paper uses a new type of ultralight and super-high thermal conductivity Pyrolytic Graphite Sheet to make a thin and flexible thermal conduction strip. Such a strip is used to design the thermal control scheme for a fully passive, modular, and fast integration of the focal plane components. Analysis and test results show that under the conditions of an installation space of $\leq 0.5 \mathrm{~mm}$ and the mass of the thermal conduction strip of $\leq 20 \mathrm{~g}$, the focal plane components work continuously for $600 \mathrm{~s}$; the maximum temperature of the CMOS (Complementary Metal-Oxide-Semiconductor) sensor is $31.8^{\circ} \mathrm{C}$. Five groups of CMOS sensors had a temperature nonuniformity no higher than $1^{\circ} \mathrm{C}$. The application of the new PGS (Pyrolytic Graphite Sheet) provides a new solution for the related space thermal design.
\end{abstract}

\section{Introduction}

With the progress of mechatronic technology in recent years, microsatellite technology has been developed rapidly. Satellite clusters and networking have been widely used in the fields of resource surveying, urban planning, precision agriculture and forestry, emergency remote sensing, and other fields [1]. In the future, small and lightweight units, low costs, short development cycles, and high functional densities will become the mainstream development trends of microsatellites [2]. A certain type of microsatellite can achieve $1 \mathrm{~m}$ resolution color video and image acquisition with a mass of only $70 \mathrm{~kg}$. It is comparable with traditional satellites massing hundreds of kilograms in terms of image quality and width.

As the core component of microsatellite imaging, the focal plane is the main function of the photoelectric signal conversion, storage, transfer and output electrical signal, and so on. The CMOS sensor has very strict working environment temperature requirements. Research by Wang et al. on some CMOS sensors showed that a temperature fluctuation caused the CMOS sensor to produce thermal noise $[3,4]$. In addition, the conversion gain of the device decreased and the saturation output decreased. The dark current doubled at every $5^{\circ} \mathrm{C} \sim 8^{\circ} \mathrm{C}$, thereby reducing the performance of the camera system. Therefore, controlling the temperature of the focal plane components is one of the core steps for obtaining high-quality images.

In order to achieve high-resolution and wide-angle video image acquisition for a certain type of microsatellite, the focal plane system is designed using five CMOS sensor-splicing schemes. The total power consumption is about $70 \mathrm{~W}$, and the total imaging time per orbital period is not less than $600 \mathrm{~s}$. Due to the constraints of volume, weight, device encapsulation, and other conditions, the layout of the electronic components is compact. The signal-processing circuit board behind the CMOS sensor has to be close to the chip and occupy almost the entire area of the back of the chip, making it difficult to establish a low thermal resistance heat conduction path by conventional means. Many high-power electronic components are arranged in a very small area, and 
the time and the space for the heat generation of the devices are concentrated. As a result, local high temperature is generated in the focal plane electric box.

\section{Thermal Design Constraints}

The core idea of the thermal design of the focal plane assembly is to make full use of the existing mature technology and thermal control technology. The thermal control measures are simple and easy to implement under the premise of ensuring that the overall indicators and reliability standards are met. This is especially true for the focal plane components designed for integrating microsatellite optics, motors, and electricity, which need a modular, lightweight, low-cost, and short-cycle thermal design.

2.1. Composition of Focal Plane Components. A certain type of microsatellite high-resolution camera consists of an optical mechanism, a focal plane component, and a focusing mechanism, in which the focal component is spliced by five groups of modular CMOS sensors. The single-module power consumption is $14 \mathrm{~W}$, and the total power consumption is about $70 \mathrm{~W}$. The modules are all the same and they are interchangeable in structure, which facilitates rapid assembly, integration, and modular testing, and reduces the development cycle and cost. Therefore, for the thermal design of the microsatellite focal plane, additional consideration must be given to its modularization, resource conservation, long-term working conditions, and light and compact design requirements.

The focal plane electronics hardware uses a layered architecture (Figure 1), and it is divided into three PCBs (Printed Circuit Board). PCB 1 includes the CMOS sensors and the driver circuit, PCB 2 includes the signal-processing components, and PCB 3 includes the power driver and the interface conversion.

The statistics of the components with a power consumption greater than $0.5 \mathrm{~W}$ for each PCB are shown in the following table.

2.2. Design Input. As described in the foreword, in order to reduce the influence of the dark current generated by the temperature fluctuation on the imaging performance of the CMOS devices, we need to control the temperature of the CMOS sensors. Their thermal design input includes the following.

2.2.1. Environmental Conditions. Affected by the cold of space, the external heat flow, and the constant heat consumption of satellites, the temperature of the microsatellite cabins is generally in the range of $-15^{\circ} \mathrm{C}$ to $+50^{\circ} \mathrm{C}$. In order to ensure the imaging quality of the camera, the temperature of the camera optical structure needs to be set to $18^{\circ} \mathrm{C}$, and the focal plane component is mounted on the back of the camera using the focusing mechanism. The average temperature of the focal plane component during analysis can be set to $10^{\circ} \mathrm{C}$.

2.2.2. Internal Heat Source. The internal heat source of the focal plane component includes the CMOS sensor heat dissipation and the heating of the electronic devices. The internal

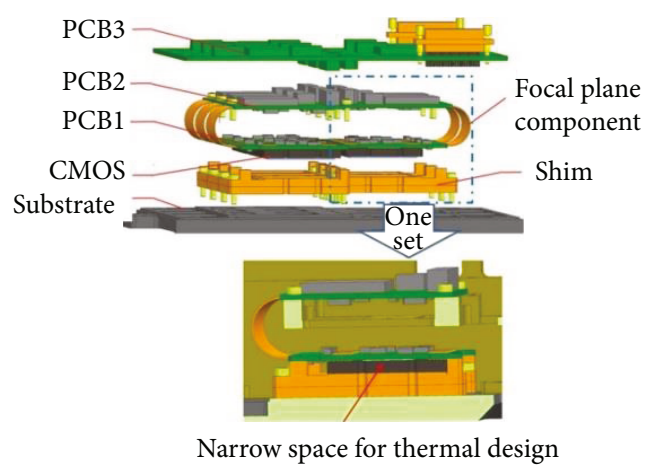

FIGURE 1: Internal structures of the focal plane components.

heat source distribution is shown in Table 1 and Figure 1. Among these components, the maximum heating powers of the main electronic devices are $2 \mathrm{~W} /$ chip for the CMOS sensor and 3.5 4 W/chip for the FPGA (Field-Programmable Gate Array) chip.

2.2.3. Functional Requirements. The size of the electrical box is $170 \mathrm{~mm} \times 160 \mathrm{~mm} \times 65 \mathrm{~mm}$ (Figure 2), with a mass of $2.3 \mathrm{~kg}$. The focal plane component can move with the focusing mechanism, and the thermal control measures should not have a risk of causing a stuck focus.

2.2.4. Thermal Design Targets. For the thermal design of the microsatellite focal plane assembly, it is required to use as few resources as possible, to achieve a rapid integration of the thermal control, to be lightweight, and to have a low cost.

Usually, the maximum temperature of the CMOS will not exceed $35^{\circ} \mathrm{C}$ for the condition of $600 \mathrm{~s}$ of imaging time per orbital period [5]. In addition, the heat generated by the focal component in an orbit period (about $5700 \mathrm{~s}$ ) can be diverted in order to achieve a temperature balance and meet the temperature control demands of a continuous multitrack long-term work. The five groups of CMOS sensors for the splicing focal planes satisfy the temperature nonuniformity condition of $\Delta \mathrm{T} \leq 1^{\circ} \mathrm{C}$ during the working time.

In space, heat is mainly dissipated by conduction, radiation, or other special means. Many scholars have systematically studied the characteristics of large-power focal plane components, and they have provided various solutions $[3,4,6-8]$. The mainstream methods are fully passive thermal control schemes and passive + active thermal control schemes. Due to the narrow thermal control space of a microsatellite, the thickness and the cross-sectional area of the heat-conducting strip are limited, and the thermal conductivity is insufficient for heat dissipation. After analyzing this method, it is apparent that it can no longer meet the heat-dissipation requirements of large-power CMOS devices.

Considering the above-mentioned design difficulties and the characteristics of microsatellites, a new type of flexible graphite film material developed in recent years [9-11] can solve the contradiction between the limited heat control resources of high power and the high thermal density, limited heat control resources, and narrow space. 
TABLE 1: Focal plane power consumption statistics.

\begin{tabular}{lccc}
\hline & Main heating device & Single set power & $\begin{array}{c}\text { Total power } \\
\text { consumption }\end{array}$ \\
\hline \multirow{2}{*}{ PCB 1 } & CMOS sensors & $2 \mathrm{~W}$ & $10 \mathrm{~W}$ \\
& Other chips & $0.5 \mathrm{~W}$ & $2.5 \mathrm{~W}$ \\
\hline \multirow{2}{*}{ PCB 2 } & FPGA-1 & $4 \mathrm{~W}$ & $20 \mathrm{~W}$ \\
& Other chips & $1 \mathrm{~W}$ & $5 \mathrm{~W}$ \\
\hline \multirow{2}{*}{ PCB 3 } & FPGA-2 & $3.5 \mathrm{~W}$ & $17.5 \mathrm{~W}$ \\
& Power drive & $2 \mathrm{~W}$ & $10 \mathrm{~W}$ \\
& Other chips & $1 \mathrm{~W}$ & $5 \mathrm{~W}$ \\
\hline \multirow{2}{*}{ Total } & - & $14 \mathrm{~W}$ & $70 \mathrm{~W}$ \\
\hline
\end{tabular}

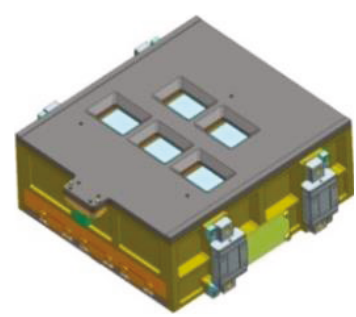

Figure 2: The focal plane electric box.

\section{Thermal Design Scheme of Focal Plane Components}

3.1. Pyrolytic Graphite Sheet [9]. PGS (Figure 3) is a kind of flexible graphite film that is a new product developed abroad to solve the heat-dissipation problem of precision electronic equipment. A graphite sheet is made from high polymer film using pyrolytic graphitization, which is completely different from the traditional process, causing the sheet to have a unique grain microstructure orientation similar to that of a single crystal of graphite. The heat can be dissipated evenly in both directions, and heat is diffused throughout the entire graphite film, thereby eliminating the local hot spots.

PGS has a smooth surface, strong flexibility, and bending resistance for more than 30,000 bends. Its main features are summarized as follows.

3.1.1. High Thermal Conductivity. PGS has the highest level of thermal conductivity in the industry. The newest product's thickness is only $10 \mu \mathrm{m}$, and its thermal conductivity is up to $1950 \mathrm{~W} /(\mathrm{m} \cdot \mathrm{K})$, which is about 10 times that of pure aluminum.

3.1.2. Light Weight. The density of PGS is in the range of $0.851 \mathrm{~g} / \mathrm{cm}^{3}-2.1 \mathrm{~g} / \mathrm{cm}^{3}$ (thickness varies with density), equivalent to $1 / 10-1 / 4$ the density of copper or $1 / 3-1 / 1.3$ the density of aluminum (the density of copper is $8.9 \mathrm{~g} / \mathrm{cm}^{3}$ and the density of aluminum is $2.7 \mathrm{~g} / \mathrm{cm}^{3}$ ).

3.1.3. Easy Processing. The graphite film has the qualities of a small weight, low thermal resistance, softness, easy processing, and repeated bending, and its lamellar structure can adapt to any surface.

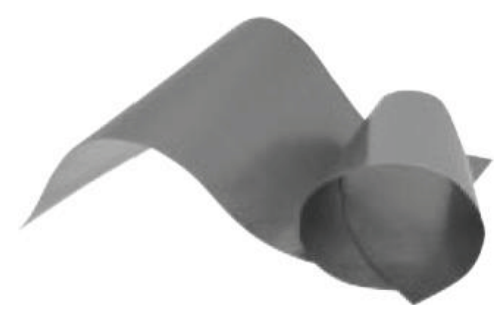

Figure 3: Pyrolytic Graphite Sheet (PGS).

Based on the above characteristics, PGS can provide more excellent thermal conductivity with less weight. It is especially suitable for lightweight space cameras with strict quality and space requirements. It provides a new approach for the thermal design of aerospace large-power focal plane components.

3.2. Thermal Conductivity Test of the PGS. As a new type of high thermal conductivity material, high safety and high reliability are prerequisites for the successful application of PGS. According to the reported applications in the aerospace field, there are many cases of aerospace use, such as Hosei Nagano using PGS to design a lightweight deployable radiator [11].

Compared to traditional high thermal conductivity materials such as aluminum, copper, and silver, high thermal conductivity PGS films have superior performance (Table 2). The thickness of the PGS film to be used in this paper is $25 \mu \mathrm{m}$, and the thermal conductivity in the plane direction can reach $1600 \mathrm{~W} /(\mathrm{m} \cdot \mathrm{K})$.

In order to verify the thermal properties of the material, this study made use of three kinds of materials, listed in Table 2, to make the same heat conduction strip, and a thermal analysis simulation and a qualitative test of their heat conduction properties were carried out.

All three types of material heat-conducting strips were $20 \mathrm{~mm} \times 220 \mathrm{~mm}$ in size and $0.1 \mathrm{~mm}$ in thickness (four layers of the PGS film are pasted together). The heat source power was $6 \mathrm{~W}$, and the heat source was located at one end of the heat-conducting strip. The results of the analysis under natural air convection at room temperature are shown in Figure 4.

Figure 4(a) shows the temperature curves near the heat source of the heat conduction strip. It can be seen that using the PGS film can greatly reduce the equilibrium temperature of the heat source and utilize its high thermal conductivity to quickly transfer heat from the high temperature area to the low temperature area, so that the temperature difference between the two ends becomes smaller and the temperature is homogenized. The equilibrium temperatures for the three kinds of material heat conduction strips were aluminum $\left(66^{\circ} \mathrm{C}\right)>$ copper $\left(62^{\circ} \mathrm{C}\right)>$ PGS $\left(44^{\circ} \mathrm{C}\right)$, which reflect the thermal conductivity characteristics.

Figure 4(b) shows the temperature curves far away from the heating source. It can be seen that after the heat source started working, the PGS film could transfer heat to the cold end more quickly. Therefore, in the early stage of heating, the PGS cold-end temperature rose faster and the final equilibrium temperature was the highest. 
TABLE 2: Comparison of different material parameters.

\begin{tabular}{lcc}
\hline Materials & Thermal conductivity $(\mathrm{W} /(\mathrm{m} \cdot \mathrm{K}))$ & Density $\left(\mathrm{kg} / \mathrm{m}^{3}\right)$ \\
\hline PGS $(25 \mu \mathrm{m})$ & 1600 & 1900 \\
Aluminum & 137 & 2700 \\
Copper & 385 & 8960 \\
\hline
\end{tabular}

The qualitative thermal conductivity test method was as follows. One end (simulated heat source) of the PGS, copper, and aluminum heat-conducting strips of the same size was affixed to the heating belt with thermal conductive silicon rubber, and the other end (the heating end) was pasted to a thermocouple. The heating power of the heating belt was $6 \mathrm{~W}$ (Figure 5).

From Figure 6, it is known that the actual test results and the software analysis results have some numerical errors due to the inaccurate simulation of the boundary conditions, the incomplete uniform thickness of the three materials, and the uneven heat source. However, the trend of the measured temperature curve of the overall measurement is consistent with the analysis, which is in turn consistent with the conclusions drawn from the previous article and qualitatively proves that the thermal resistance of the PGS thin film is small.

3.3. Thermal Design of Focal Plane. Based on the characteristics of excellent thermal conductivity and the light weight of PGS thin films, this study developed a thermal design for microsatellite large-power focal plane components. The design adopted fully passive thermal control measures in order to divert the large heat flow at the heat source to a larger heat capacity and temporarily store it, and then slowly dissipate it through conduction and radiation.

3.3.1. Structural Design. In order to optimize the heat-dissipation effect of the PCB-heating devices, a structural framework was designed based on the rational layout of the PCB and the heat dissipation of copper. The focal plane-splicing substrate adopted a titanium alloy that was matched with the thermal expansion coefficient of the chip material, and an aluminum alloy was used for the electric box frame.

3.3.2. Thermal Radiation Isolation. A layer of $2 \mathrm{~mm}$ thick thermal insulation board was added between the focal plane imaging module and the signal-processing module (between PCB 1 and PCB 2), to isolate the influence of the temperature rise in the CMOS sensor when the power module worked.

3.3.3. Heat Dissipation. A heat-conducting strip made of four layers of PGS $(25 \mu \mathrm{m})$ was attached to the back of each CMOS sensor, and the total thickness was $0.1 \mathrm{~mm}$ to $0.15 \mathrm{~mm}$. Both ends of the heat-conducting strip were bent and pasted onto the heat-insulating aluminum frame (Figure 7). Through the high thermal conductivity path, the concentrated heat generated by the CMOS focal plane was dredged to the framework of the electric box structure to eliminate hot spots and avoid thermal stress concentrations.
Modular thermal design is conducive to the replacement, rapid integration, and maintenance testing of a single group focal plane component.

The main heat-dissipation path design of a large-power CMOS sensor is shown in Figure 8, where the PGS heat conduction strip is the main heat-dissipation path. Most of the heat generated by the focal plane component is directed outward through the heat-conducting strip, the radiator of the electric box, the cooling surface of the satellite cabin, and the cold of space.

It is important to note that PGS films need special handling when they are being tailored. The PGS film of the original package had the composition structure shown in Figure 9. The middle layer was a graphite heat-conducting layer made of pyrolytic graphitization. Its texture was soft and easy to break off. The upper surface was a high heat-resistant PEEK (polyetheretherketone) protective film, and the lower surface was a B-type acrylic double-sided adhesive. The upper and lower surfaces played the role of supporting and shaping, and the edges were protected by the edge seal to prevent the graphite layer from dropping the slag.

In actual use, cutting is unavoidable in order to obtain a specific shape, the edge seal may be damaged, and the graphite slag may fall into the circuit board, the pins, the photosensitive surface, or other areas to cause contamination. Therefore, after cutting, special measures are needed to reseal the protective layer and prevent accidents.

\section{Thermal Analysis of the Focal Plane Electric Box}

In this study, a series of simplifications of the physical model of the focal plane were performed, and a simulation analysis was performed using the Thermal Desktop analysis software. This was done in order to verify the effect of the thermal design scheme of the large-power focal plane component, shorten the product development cycle, and improve the rationality and reliability of the design.

4.1. Model Simplification. Ignoring the details of electrical connectors, wires, screws, etc., the model was properly simplified; each cell was viewed in the thermal model as an isothermal body, represented by a node.

Since the focusing mechanism was a moving mechanism, there was a gap present, so the thermal conduction of the focusing mechanism was not considered in the thermal analysis process.

4.2. Thermal Analysis Results. The simplified thermal analysis model and the temperature distribution diagram are shown in Figure 10. During the analysis, the ambient temperature was maintained at $10^{\circ} \mathrm{C}$ and the initial temperature of all of the nodes was $10^{\circ} \mathrm{C}$.

The focus plane component was subjected to transient thermal analysis under initial boundary conditions to verify various design targets such as the temperature of the focal plane of the CMOS sensors, the uniformity of multiple conditions of the splicing temperatures, and the temperature balance capability of one orbital period. 


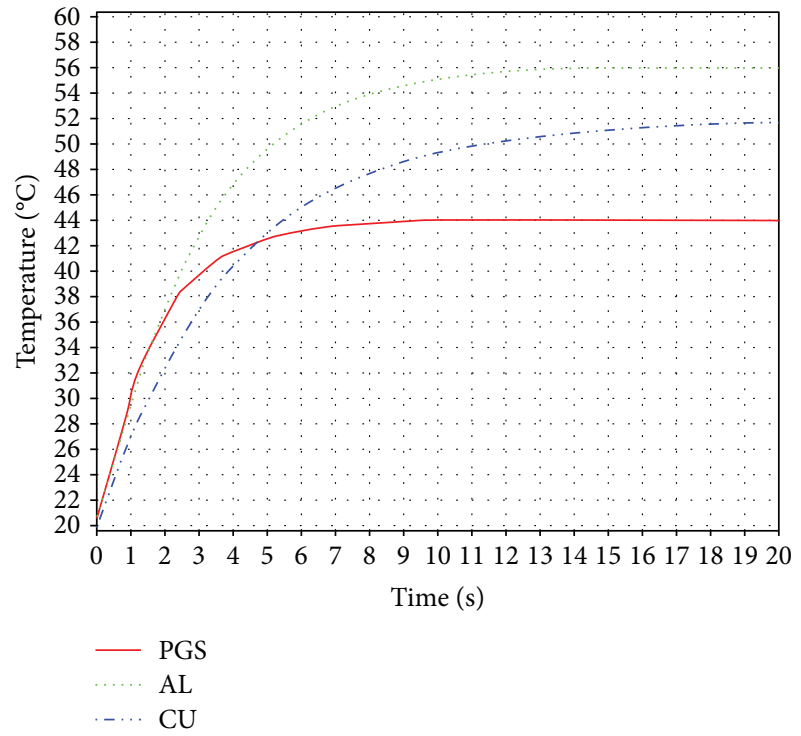

(a) Temperature near the heating source

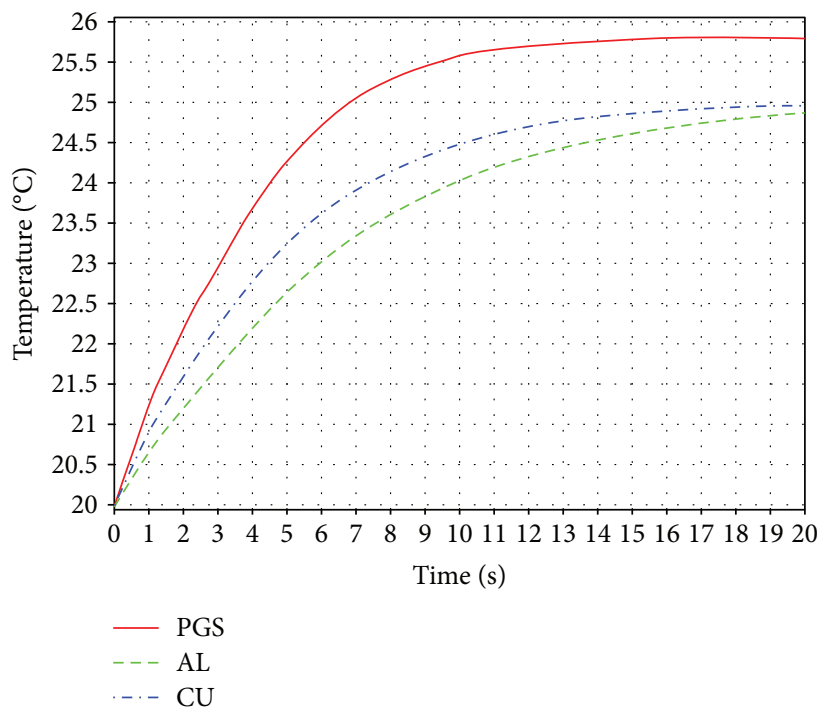

(b) Temperature far away from the heating source

FIgURE 4: Comparison of the different materials.

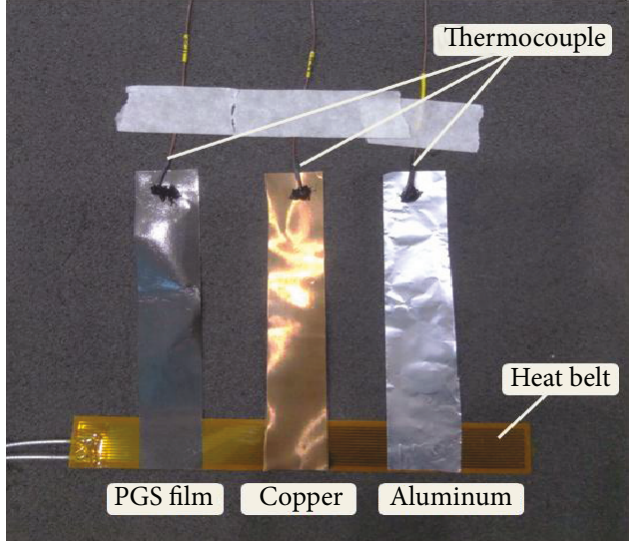

FIGURE 5: Qualitative test of thermal conductivity.

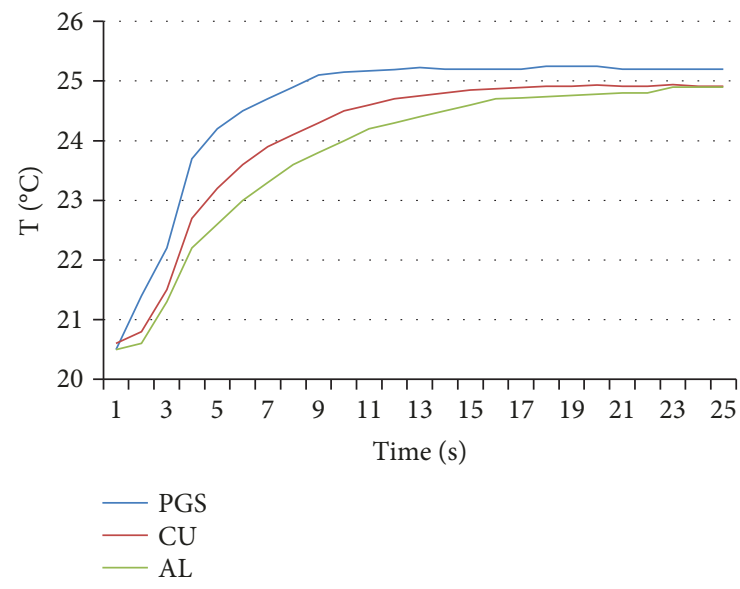

FIgURE 6: Temperature variation curves of the test.

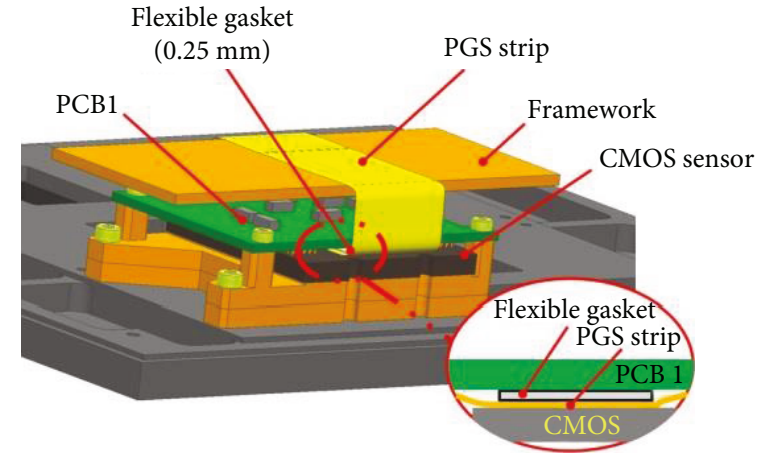

FIgURe 7: Heat-dissipation path.

The peak temperatures of the large-power components are shown in Table 3 and Figure 11, in which the maximum temperature of the CMOS sensor is $34.2^{\circ} \mathrm{C}$, which is better than the design target. The temperature of the PGS heat conduction strip was synchronized with the temperature of the CMOS. This shows that the thermal conductivity of the heat-conducting strip was sufficient to quickly guide the heat to the frame of the electric box. The temperature of the FPGA was about $61^{\circ} \mathrm{C}$, which was high, but still within the allowable temperature range of the FPGA.

In the worst-case scenario, the focal plane would be continuously working for $600 \mathrm{~s}$. The temperature variation curves of the five CMOS sensors are shown in Figure 12. From the curves, it can be seen that the trends of temperature variation of multiple groups of splicing chips are relatively uniform, and the temperature distribution nonuniformity between each other is less than $2^{\circ} \mathrm{C}$, which satisfies the design targets.

In an orbital period (about $5700 \mathrm{~s}$ ), the temperature of the CMOS sensor falls from the highest temperature of $34.2^{\circ} \mathrm{C}$ to about $18^{\circ} \mathrm{C}$, indicating that if the heat is only radiated from 


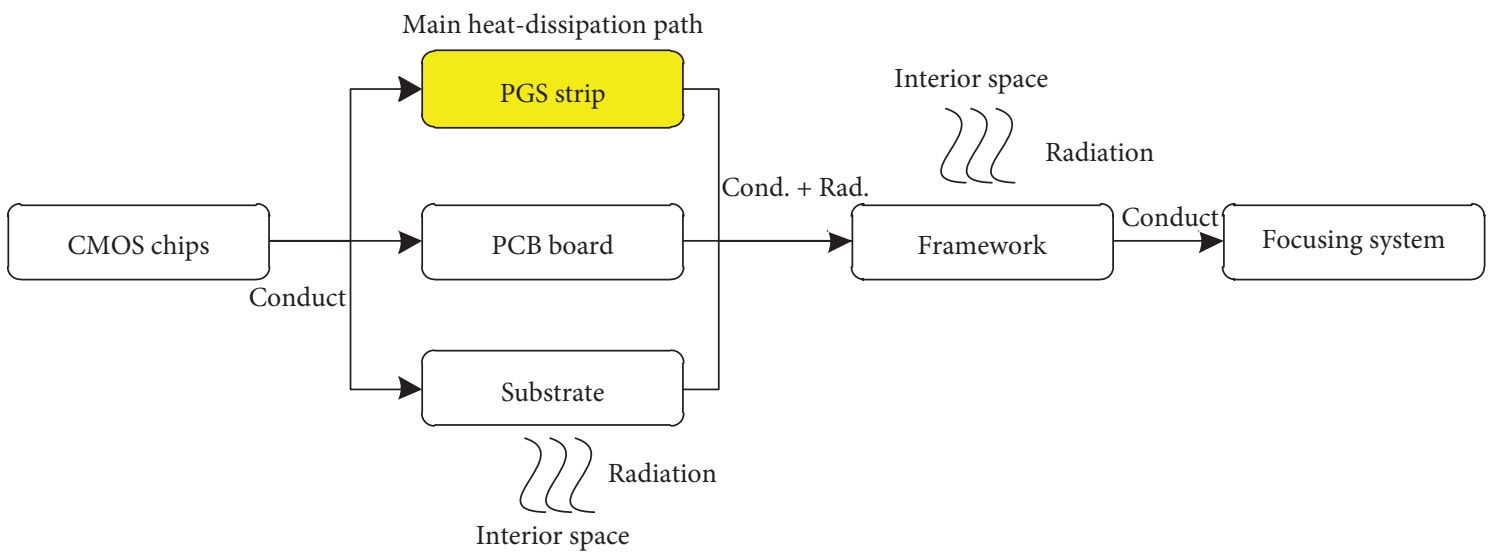

Figure 8: Design of main heat-dissipation path.

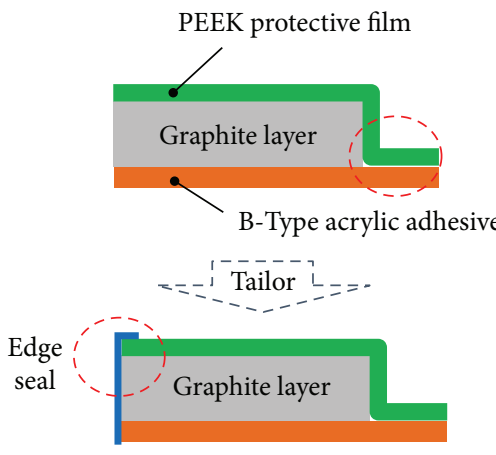

FIgure 9: The configuration of the PGS film cutting edge.

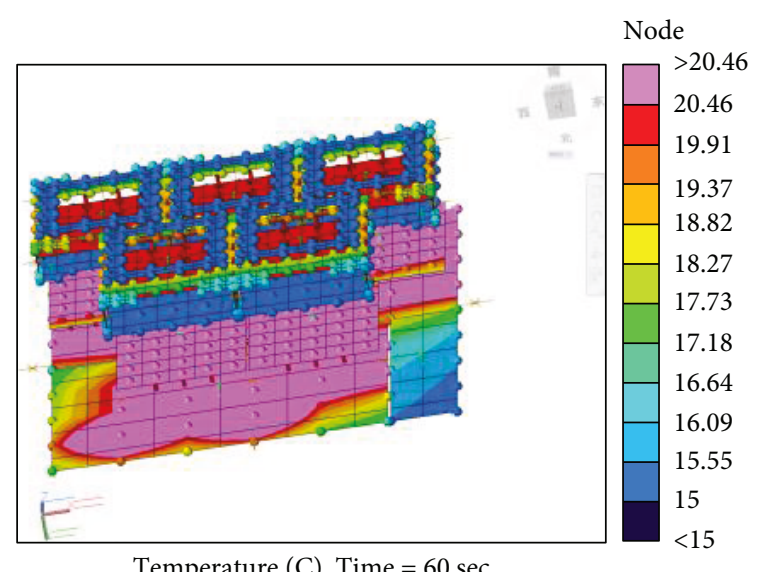

FIgURE 10: Temperature nephogram.

TABLE 3: Statistical table of characteristic temperature.

\begin{tabular}{lccc}
\hline $\begin{array}{l}\text { Large-power } \\
\text { chips }\end{array}$ & $\begin{array}{c}\text { Single set } \\
\text { power }\end{array}$ & $\begin{array}{c}\text { Peak } \\
\text { temperature }\end{array}$ & $\begin{array}{c}\text { Temperature } \\
\text { rise }\end{array}$ \\
\hline CMOS & $2 \mathrm{~W}$ & $34.2^{\circ} \mathrm{C}$ & $24.2^{\circ} \mathrm{C}$ \\
PGS strip & - & $34.2^{\circ} \mathrm{C}$ & $24.2^{\circ} \mathrm{C}$ \\
Framework & - & $30.5^{\circ} \mathrm{C}$ & $20.5^{\circ} \mathrm{C}$ \\
FPGA-1 & $4 \mathrm{~W}$ & $61^{\circ} \mathrm{C}$ & $51^{\circ} \mathrm{C}$ \\
FPGA-2 & $3.5 \mathrm{~W}$ & $50.5^{\circ} \mathrm{C}$ & $40.5^{\circ} \mathrm{C}$ \\
\hline
\end{tabular}

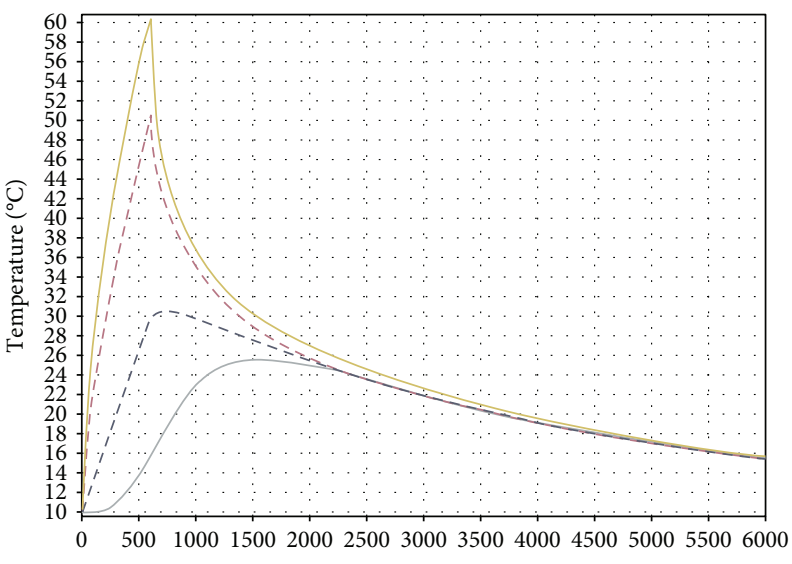

Time (s)

$$
\begin{aligned}
& \text { - - Framework - - FPGA-2 } \\
& \text { - FPGA-1 - Substrate }
\end{aligned}
$$

FIgURE 11: Characteristic temperatures.

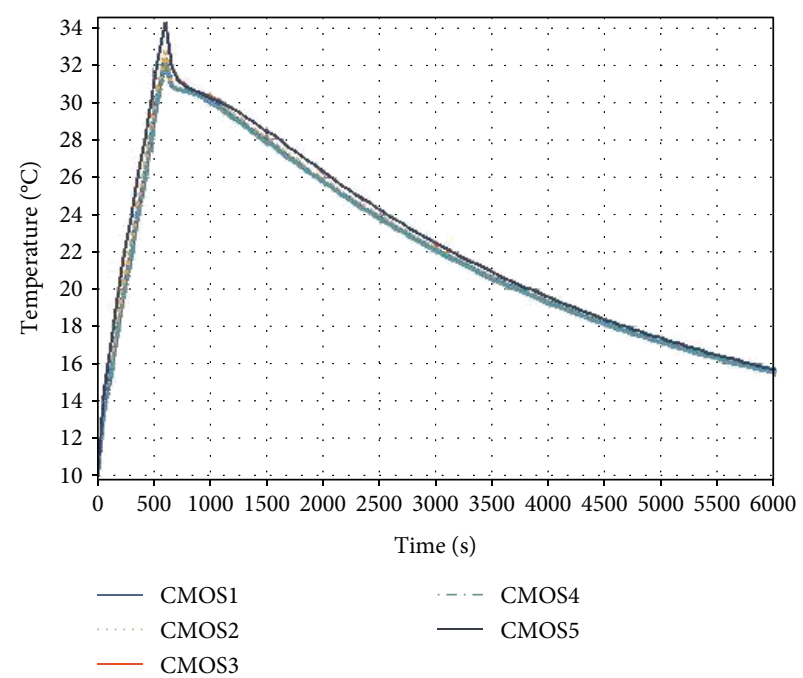

FIGURE 12: Temperature comparisons of the five groups. 
the surfaces of the electric box, the heat generated by the focal plane components cannot be totally eliminated in a single orbital period. In order to achieve a temperature balance in each orbital period, it is necessary to open the heat-dissipation surface of the satellite cabin facing the electric box of the focal plane in order to reduce the average temperature, increase the temperature difference of the heat radiation, and enhance the radiation effect. Through a joint analysis and calculation for the microsatellite, a proper design of the radiant heat-dissipation surface (with an average temperature maintained at $0^{\circ} \mathrm{C}$ ) can achieve a temperature balance in one orbital period.

In a comparative analysis of the temperature rise of a CMOS sensor based on the thermal control of a focal plane with a PGS strip and the absence of thermal control measures (Table 4), it can be seen that if there is no thermal control measure, the temperature of a CMOS sensor can reach $51.5^{\circ} \mathrm{C}$, which is far beyond the allowable temperature. Using the heat control measures of high thermal conductivity in a narrow installation space, under the condition of a mass of $20 \mathrm{~g}$, the peak temperature dropped by $17.3^{\circ} \mathrm{C}$, realizing the targets of high efficiency, low cost, and modularized thermal design for the microsatellites.

\section{Heat Test for the Focal Plane}

In order to verify the correctness of the design analysis and the actual use of the PGS heat strip, the thermal control of the focal plane electrical box was tested. Under the condition of thermal vacuum test, the in-orbit working state was simulated and the temperature rise of the CMOS sensor was measured under continuous working conditions for $600 \mathrm{~s}$.

The test system is composed of the focal plane electric box, the electric box power supply, the temperature measurement inspection instrument, the temperature sensor (T-type thermocouple), and the vacuum tank (Figure 13).

The test methods and processes include the following:

(1) Randomly selecting two CMOS sensors and pasting the T-type thermocouples behind them. Pasting a thermocouple on the PGS film at the same time, connecting the cable, and debugging the imaging system and temperature measurement system

(2) Vacuuming, starting to collect the temperature data, and waiting for the temperature balance

(3) Simulating the on-orbit work status, starting the work of the focal plane electric box, setting the acquisition cycle to $5 \mathrm{~s}$, and recording the temperature changes at each temperature measurement point

(4) The focal plane electric box is then shut down, and the temperature is continuously collected. The time required for temperature recovery is recorded

The photo of the test site is shown in Figure 14, and the temperature rise curves at each point are shown in Figure 15. From the test results, we can see that the highest
TABLE 4: Comparison of the control measures.

\begin{tabular}{lcc}
\hline Thermal control measures & $\begin{array}{c}\text { Peak } \\
\text { temperature }\end{array}$ & $\begin{array}{c}\text { After one orbital } \\
\text { period }\end{array}$ \\
\hline With PGS strip & $34.2^{\circ} \mathrm{C}$ & $18^{\circ} \mathrm{C}$ \\
Without heat control & $51.5^{\circ} \mathrm{C}$ & $29.3^{\circ} \mathrm{C}$ \\
\hline
\end{tabular}

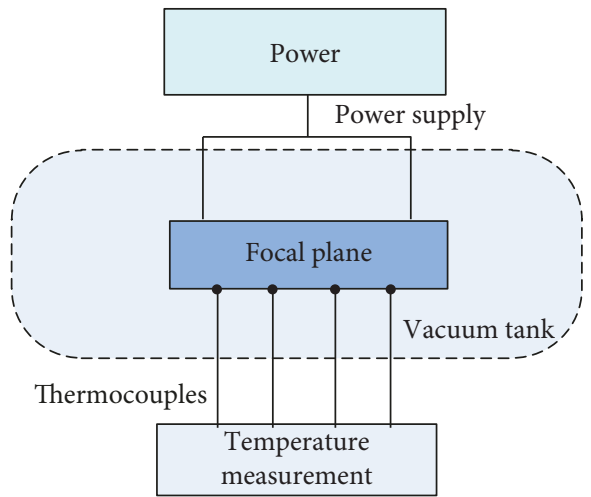

Figure 13: Test schematic diagram.

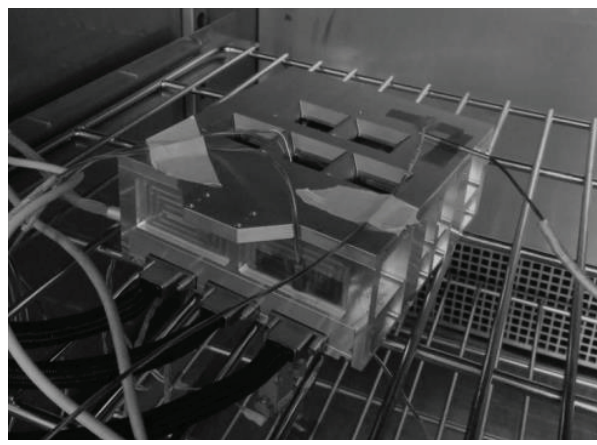

Figure 14: Thermal test condition of the focal plane.

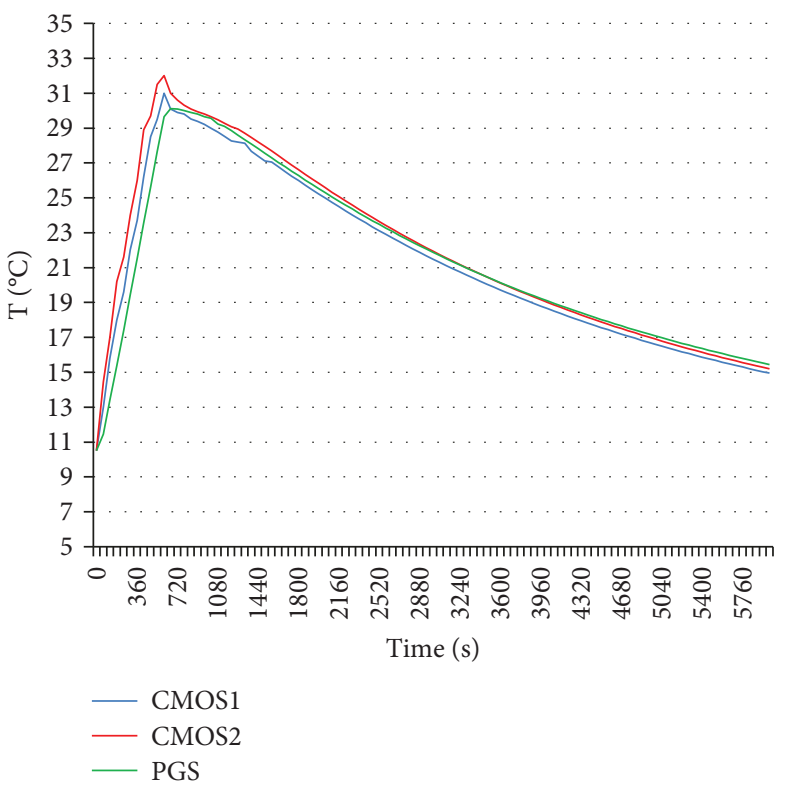

Figure 15: Thermal test temperature curves. 
temperature of the CMOS was $31.8^{\circ} \mathrm{C}$, and the temperature of the PGS heat-conducting strip was essentially the same as that of the chip. This indicates that the thermal conductivity of the flexible PGS film was very high, and that it could quickly transfer heat to the frame of the electric box. The maximum temperature difference between the two groups of CMOS was less than $1^{\circ} \mathrm{C}$, which indicates that the CMOS sensor had a better temperature consistency.

It is worth noting that at an ambient temperature of $10^{\circ} \mathrm{C}$, the temperature level of the focal plane returned to about $15.5^{\circ} \mathrm{C}$ in one orbit cycle, which was a specific distance from the design target. Therefore, a follow-up needs to be jointly designed with the satellite in order to further reduce the ambient temperature and achieve a temperature balance in each orbital period.

Through the test and analysis, the overall trends of the test results and the thermal analysis results are consistent, but there is a certain numerical deviation, and the maximum temperature deviation is close to $3^{\circ} \mathrm{C}$. The main reason for this is presumed to be that there are deviations in the values of the contact resistance and the model simplification. From this, it can be seen that the test results are consistent with the analysis results, which proves that the thermal design and the thermal simulation of large-power focal plane components based on the PGS thermal conductivity strips are reasonable.

\section{Conclusion}

A new ultrathin and ultrahigh thermal conductivity PGS film is used in this study in order to solve the heat-dissipation problems of certain types of microsatellite focal plane components with the conditions of limited resources and a narrow space and ensure the imaging quality of CMOS sensors. This is suitable for cases where the traditional heat control measures are difficult to use to meet the temperature requirements. The thickness of the selected PGS film was $25 \mu \mathrm{m}$, and the thermal conductivity in the plane direction reached $1600 \mathrm{~W} /(\mathrm{m} \cdot \mathrm{K})$. Through a modeling analysis and a qualitative comparison test of the heat conduction strip, it was found that it had a faster thermal conductivity than the traditional metal heat conduction strip, and the heat source temperature could be reduced by about $20^{\circ} \mathrm{C}$ under the prevalent conditions.

A thermal control scheme based on a PGS thermal strip for the passive, modular, and fast integration of large-power focal plane components was designed in this paper. By establishing a heat conduction path with a low thermal resistance, we used the large capacity of the electric box frame to eliminate the hot spots of the CMOS sensors. Subsequently, a thermal analysis model was established. After a thermal simulation analysis and a thermal vacuum test verification, the following results were shown.

Under the conditions of an installation space of $\leq 0.5 \mathrm{~mm}$, a mass of thermal conduction strip of $\leq 20 \mathrm{~g}$, and focal plane components working continuously for $600 \mathrm{~s}$, the maximum temperature of the CMOS sensor was $31.8^{\circ} \mathrm{C}$, and five groups of CMOS sensors had a temperature nonuniformity no higher than $1^{\circ} \mathrm{C}$. This shows that the design of the thermal control system is reasonable, the thermal control measures adopted can meet the design requirements, and the targets of high efficiency, low cost, and modular thermal design for microsatellites have been achieved.

The test and application cases of new PGS materials provide new design ideas and a reference basis for related areas of space thermal design.

\section{Data Availability}

The data used to support the findings of this study are available from the corresponding author upon request.

\section{Conflicts of Interest}

The authors declare that there are no conflicts of interest regarding the publication of this paper.

\section{Acknowledgments}

This work has been supported by the National Natural Science Foundation of China (Grant No. 51705497) and Science and Technology Development Program of Jilin (No. 20170101164JC).

\section{References}

[1] J. P. Yu, G. Q. Yang, and X. W. Liang, Modern Small Satellite Technology and Application, Shanghai Science PopuLarization Press, Shanghai, China, 2004.

[2] S. Khorram, S. A. C. Nelson, F. H. Koch, and C. F. van der Wiele, "Remote sensing," in SpringerBriefs in Space Development, pp. 2682-2698, Springer, Berlin, 2012.

[3] Y. D. LI, B. Wang, Q. Guo, M. Li-Ya, and R. Jian-Wei, “Testing system for radiation effects of CCD and CMOS image sensors," Optics and Precision Engineering, vol. 21, no. 11, pp. 2778-2784, 2013.

[4] F. Wang, G. Qi, W. Bo, and Z. Xing-Yao, "Temperature effects on performance parameters in $4 \mathrm{~T}$ CMOS image sensor," Chinese Journal of Luminescence, vol. 37, no. 3, pp. 332-337, 2016.

[5] L. H. Chen, Y. C. Li, Z. T. Luo, J. H. Dong, Z. S. Wang, and S. Y. Xu, "Thermal design and testing of CCD for space camera," Optics and Precision Engineering, vol. 19, no. 9, pp. 2117-2122, 2011.

[6] G. Q. Li and G. H. Yao, "Thermal analysis and thermal balance test of CCD assembly," Spacecraft Recovery \& Remote Sensing, vol. 24, no. 3, pp. 15-18, 2003.

[7] X. H. Lian, W. Q. Wang, Q. L. Huang et al., "Simulation test on improved thermal design of remote sensor's focal plane circuit," Spacecraft Recovery \& Remote Sensing, vol. 33, no. 4, pp. 50-56, 2012.

[8] L. H. Chen, Q. W. Wu, Z. T. Luo, J. H. Dong, and F. Jiang, "Design for thermal control system of electronic equipment in space camera," Optics and Precision Engineering, vol. 17, no. 9, pp. 2145-2152, 2009.

[9] E. Silverman, Panasonic's Thermally Conductive Pyrolytic Graphite Sheet (PGS), Panasonic Industrial Company Product Management Department, 2005. 
[10] H. Nagano, A. Ohnishi, and Y. Nagasaka, "Development of a lightweight deployable/stowable radiator for interplanetary exploration," Applied Thermal Engineering, vol. 31, no. 16, pp. 3322-3331, 2011.

[11] R. Karam, Satellite Thermal Control for Systems Engineers, American Institute of Aeronautics and Astronautics, 2015. 


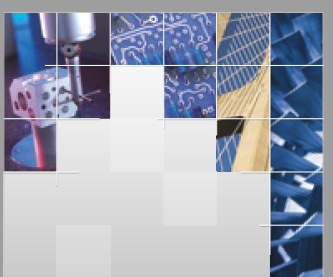

\section{Enfincering}
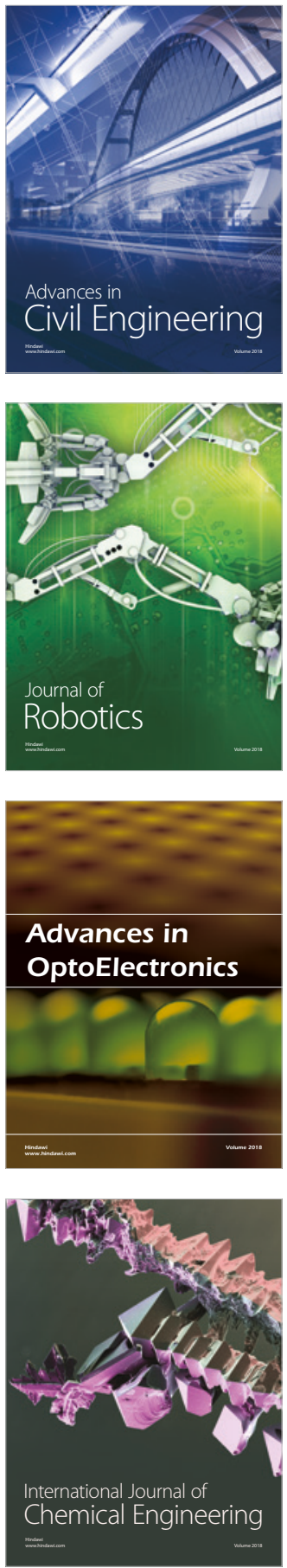

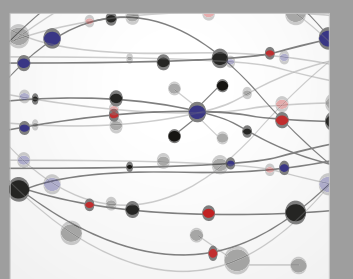

\section{Rotating \\ Machinery}

The Scientific World Journal

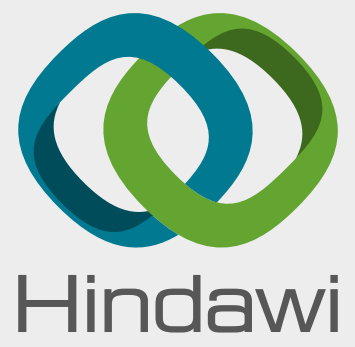

Submit your manuscripts at

www.hindawi.com
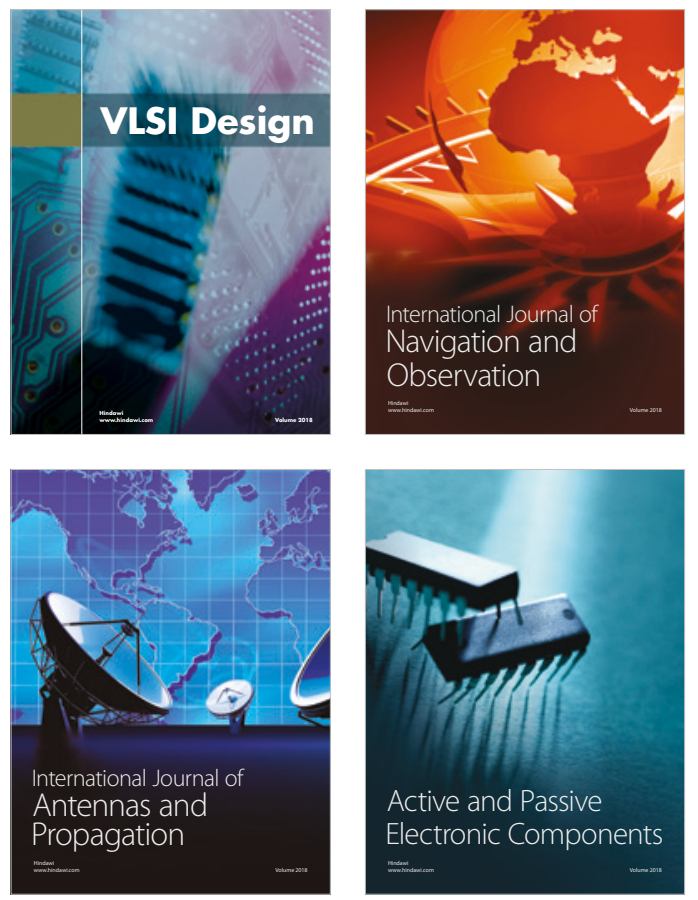
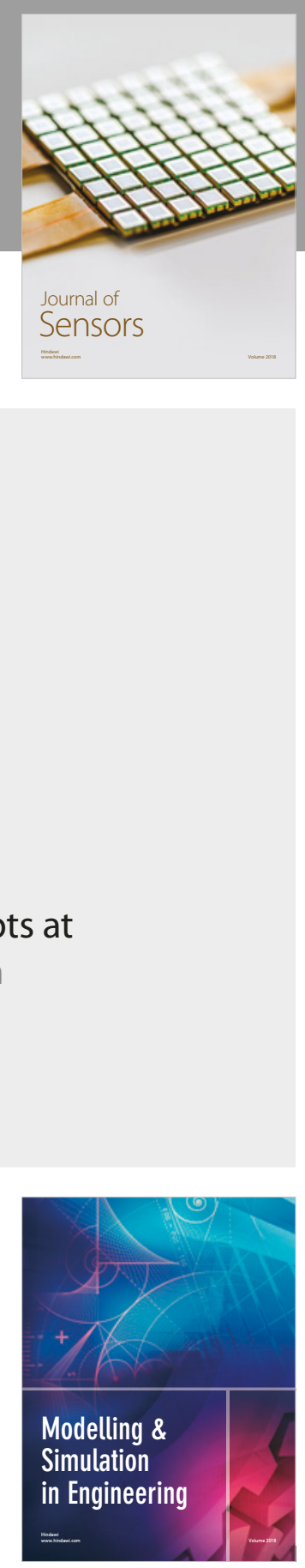

\section{Advances \\ Multimedia}
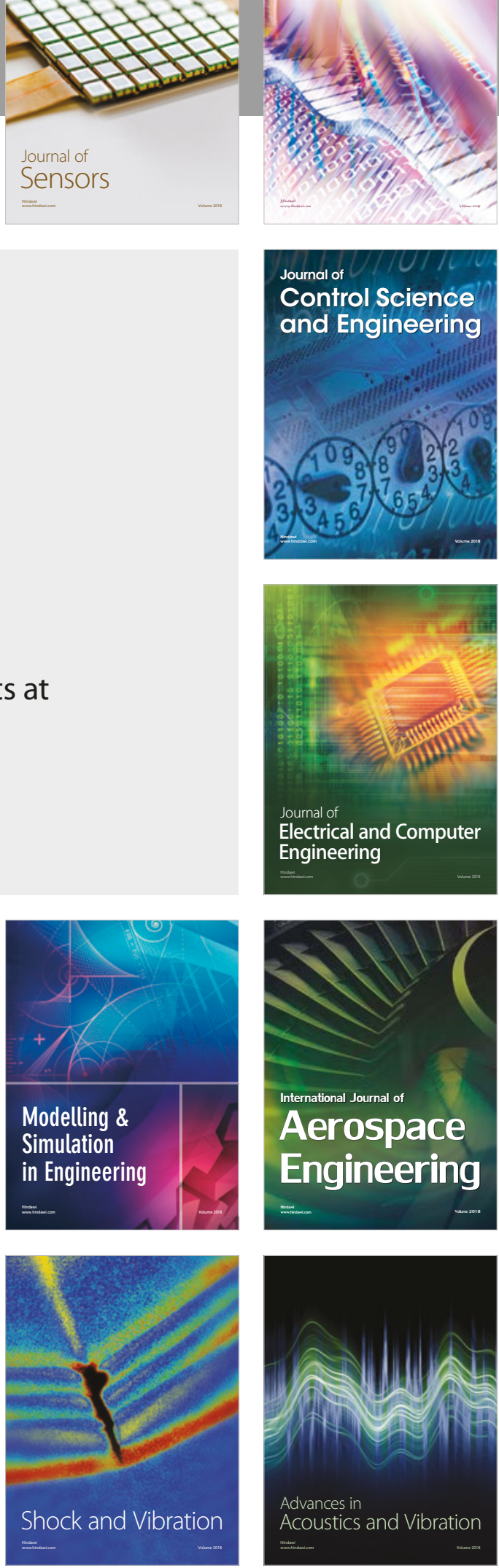